\title{
Covid-19: UK ramps up testing by $500 \%$ as health minister tests positive for virus
}

\author{
Elisabeth Mahase
}

The BMJ

UK health minister Nadine Dorries has tested positive for covid-19 and is self-isolating at home-as is Labour MP Rachael Maskell, who met with Dorries. The Department of Health and Social Care said that Dorries started showing symptoms on 5 March. As of 11 March, 456 cases of the virus have been confirmed in the UK, with six deaths.

Bharat Pankhania, senior clinical lecturer at the University of Exeter Medical School, said, "The example of Nadine Dorries getting infected, developing symptoms, and then realising a few days later that she had acquired the coronavirus infection demonstrates the difficulty of controlling the spread of the coronavirus in the community."

The news of the first UK MP infected with the virus came as the government announced that it would be substantially expanding testing, with Public Health England's test set to be rolled out to regional laboratories across the country. NHS England said that this would enable 10000 tests to be carried out daily, compared with the 1500 tests that are currently processed every day at Public Health England laboratories. As of 10 March, 25000 tests had been completed.

The government said that it was "scaling up tests by $500 \%$ " to ensure capacity as more people come forward. To do this, NHS England has asked NHS laboratory services across the country to "bring new capacity online, and other labs to begin checks, enabling 8000 more samples to be analysed every day of the week."

Jo Martin, president of the Royal College of Pathologists, said: "Scaling up pathology testing fivefold for covid-19 is a considerable ask of our virology and infection experts, but they are already rising to these challenges. They have been working non-stop to support patients and the public in this. They have been flexible and applying their considerable skills in this vital area, and we will do what we can to help them with further support as the situation develops."

Will Irving, professor of virology at the University of Nottingham, told The BMJ that his laboratory had been asked to take on 250 tests a day on top of their usual workload. He said that, although there might be "enough slack in the system" to do this, it could mean that they have to defer doing other tests. Irving said that he was concerned about staffing in the case of school closures or strict self-isolation.

The Public Health England test for covid-19 includes a nose or throat swab that scrapes cells from these areas. The genetic material is extracted from these swabs and tested using a polymerase chain reaction test to determine whether genetic material from the virus - which was sequenced early on during the initial outbreak in China-is present. 